\title{
Synergistic Effect of Fluorinated Passivator and Hole Transport Dopant Enables Efficient and Stable Perovskite Solar Cells with Efficiency Near 24\%
}

Hongwei Zhu ${ }^{1,2,3}$, Yameng Ren ${ }^{* 2}$, Linfeng Pan $^{4}$, Olivier Ouellette ${ }^{2}$, Felix T. Eickemeyer ${ }^{2}$, Yinghui $\mathrm{Wu}^{4}$, Xianggao $\mathrm{Li}^{* 1,3}$, Shirong Wang ${ }^{1,3}$, Hongli $\mathrm{Liu}^{1,3}$, Xiaofei Dong ${ }^{1,3}$, Shaik M. Zakeeruddin ${ }^{2}$, Yuhang Liu $^{* 2}$, Anders Hagfeldt ${ }^{4}$, and Michael Grätzel ${ }^{* 2}$

\section{Tianjin University, School of Chemical Engineering and Technology, Tianjin 300072, China}

2. Laboratory of Photonics and Interfaces (LPI), Department of Chemistry and Chemical Engineering, École Polytechnique Fédérale de Lausanne, Lausanne CH-1015, Switzerland.

3. Collaborative Innovation Center of Chemical Science and Engineering, Tianjin 300072, China

4. Laboratory of Photomolecular Science (LSPM), École Polytechnique Fédérale de Lausanne, Station 6, CH-1015 Lausanne, Switzerland.

${ }^{\dagger}$ Corresponding to: Dr. Yuhang Liu, yuhang.liu@epfl.ch, Dr Yameng Ren, yameng.ren@epfl.ch, Prof. Xianggao Li, lixianggao@tju.edu.cn, Prof. Michael Graetzel, michael.graetzel@epfl.ch.

\begin{abstract}
Long-term durability is critically important for the commercialization of the advanced perovskite solar cell (PSC) technology. Since the ionic perovskite materials and the hole-transporting layer (HTL) additives customarily used, for example, lithium bis(trifluoromethanesulfonyl)imide (LiTFSI) and tert-butyl pyridine( $t \mathrm{BP})$, are intrinsically hydrophilic and the degradation of the PSCs upon moisture uptake compromises long-term stability of the PSCs, it is important to take steps to reduce their hydrophilicity. In this work, we employ a trifluoromethylation strategy to chemically functionalize the perovskite passivation layer and the HTL additive, to yield 4-trifluoromethylbenzylammonium iodide (FBAI) as a molecular passivator and 4-(trifluoromethyl)pyridine (TFP) as a HTL additive, respectively. Surface treatment of the triple cation perovskite $\left(\mathrm{Cs}_{0.05} \mathrm{FA}_{0.85} \mathrm{MA}_{0.10} \mathrm{~Pb}\left(\mathrm{I}_{0.97} \mathrm{Br}_{0.03}\right)_{3}\right)$ light harvester with FBAI largely suppressed the nonradiative charge carrier recombination, boosting the power conversion efficiency (PCE) from $20.9 \%$ to $23.9 \%$ with negligible hysteresis. Adding TFP into the HTL yielded strong resistance to moisture for the PSC while similar PCE compared to the standard HTL PSC devices was maintained. When
\end{abstract}


combining FBAI surface passivation with adding TFP into the HTL, our new synergistic approach successfully increased the PSC device's hydrophobicity through fluorination of both the HTL additive and the passivation material, leading to FBAI/TFP based PSCs retaining over $96 \%$ of their initial efficiency after $500 \mathrm{~h}$ maximum power point tracking (MPPT) under simulated full-sun irradiation, and $97 \%$ of their initial efficiency after $1100 \mathrm{~h}$ storage under ambient condition at a relative humidity (r.h.) of $60-70 \%$.

\section{Introduction}

During the past decade the development of perovskite solar cells (PSCs) has become a gamechanger in the field of photovoltaics with relatively rapid progress in the attainment of power conversion efficiencies (PCEs) of over $25 \%{ }^{1-5}$. Along with the fundamental breakthroughs, the commercialization of PSCs has been advanced. Improvement in the stability of PSC devices remains the most challenging issue ${ }^{6-10}$ to this PV technology. The best performing PSCs are mostly based on a $\mathrm{n}-i$-p device structure, where $\mathrm{n}$ refers to $n$-type semiconductor, normally $\mathrm{TiO}_{2}{ }^{11 ; 12}$ or $\mathrm{SnO}_{2}{ }^{13 ; 14}$. The organic-inorganic lead halide perovskite used as the light-absorbing layer is sandwiched between the electron transporting layer (ETL) and the hole transporting layer (HTL) for selective charge carrier extraction. Since the ETL is deposited in-between the transparent conductive substrate (e.g. fluorine doped tin oxide (FTO) or indium doped tin oxide (ITO).) and the perovskite active layer, water and oxygen ingression from the environment destroys the PSCs mainly at the perovskite/HTL interface ${ }^{15 ; 16}$. Ambient moisture uptake is one of the key issues resulting in poor operational stability of the PSCs due to the intrinsic hydrophilicity of the perovskite active layer materials ${ }^{17 ;}$ 18. In addition, the widely used HTL additives, such as (lithium bis(trifluoromethanesulfonyl)imide (Li-TFSI) and tert-butyl pyridine $(t \mathrm{BP})$ ), are hydrophilic, intensifying moisture penetration from ambient air to the perovskite through the HTL ${ }^{19-22}$. Liu et $\mathrm{al}^{23}$ reported that the use of fluorinated phenylethylammonium iodide can greatly enhanced $i$ the hydrophobicity of the perovskite, improving operational stability against moisture. However, the effect of the hydrophobic HTL additives on the PCE and the long term stability has remained less explored.

In this work, we firstly applied a novel trifluoromethylated interface passivation material, 4- 
trifluoromethyl-benzylammonium iodide (FBAI), to augment the hydrophobicity of the $\mathrm{Cs}_{0.05} \mathrm{FA}_{0.85} \mathrm{MA}_{0.10} \mathrm{~Pb}\left(\mathrm{I}_{0.97} \mathrm{Br}_{0.03}\right)_{3}$ triple cation perovskite while simultaneously suppressing the defects. The perovskite film passivated with FBAI exhibits a much higher photoluminescence quantum yield (PLQY) and lower $V_{\mathrm{OC}}$ loss against the radiative limit compared with the pristine film (Figure 3d), revealing the excellent passivation effect of FBAI. Then, in order to increase the hydrophobicity of the triple cation perovskite active layer and the HTL layer while maintaining the high efficiency of the spiro-OMeTAD/tBP system, we modified spiro-OMeTAD HTL by adding the fluorinated additive, TFP. Time-resolved photoluminescence (TRPL) measurements and external photoluminescence quantum efficiencies (PLQY ${ }_{\text {ext }}$ ) show that the introduction of TFP does not alter the extraction of holes and recombination processes at perovskite/spiro-OMeTAD interface (Figure S8 and S9). A champion PSC device based on perovskite/FBAI/(spiro-OMTAD/TFP) obtained an outstanding $V_{\mathrm{OC}}$ of $1.163 \mathrm{eV}$, with a low voltage loss of $387 \mathrm{mV}$. Remarkably, FBAI/TFP based PSCs retain over $96 \%$ of their initial efficiency after $500 \mathrm{~h}$ maximum power point tracking (MPPT) under simulated full-sun irradiation and $97 \%$ of their initial efficiency after $1100 \mathrm{~h}$ under ambient condition at a relative humidity (r.h.) of $60-70 \%$.

\section{Results and discussion}

a

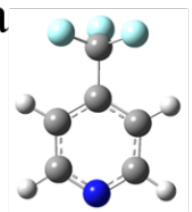

TFP

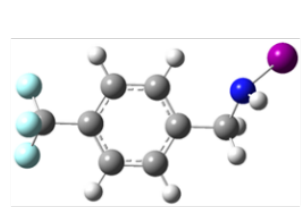

FBAI
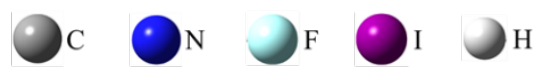

$\mathrm{C}$

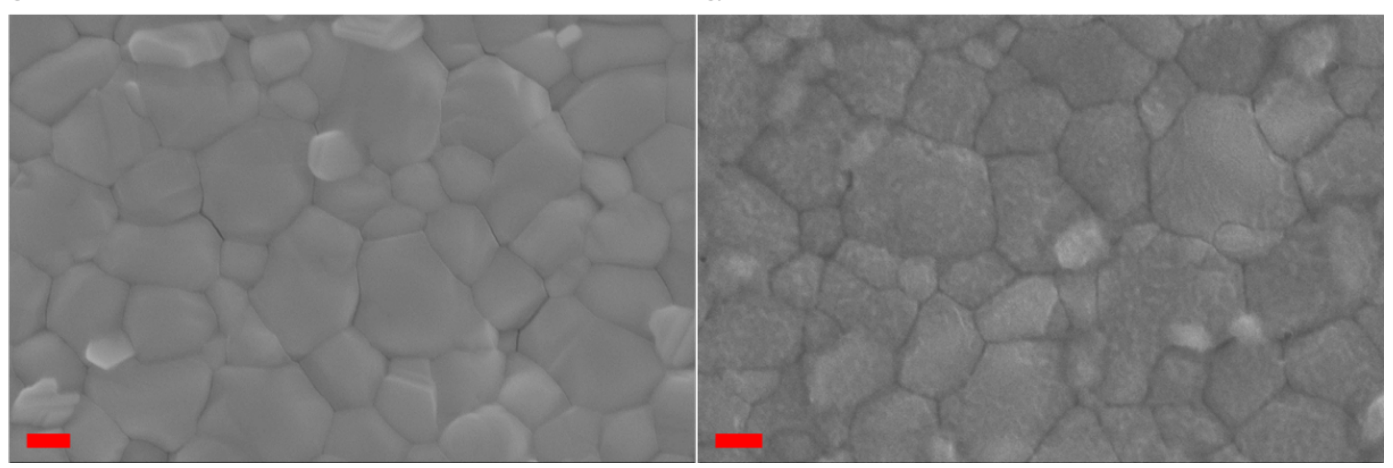

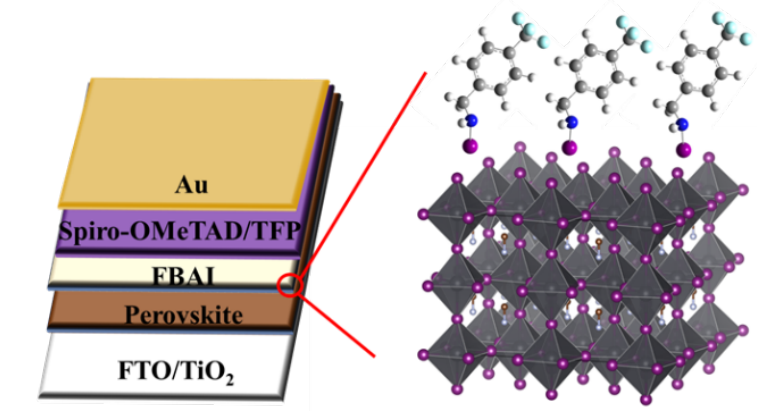

d 
Figure 1. a) Chemical structures of TFP and FBAI. b) Structures of a FBAI/TFP based PSC, c) and d), SEM images of a neat perovskite film, and a perovskite film passivated by FBAI. The scale bar is $200 \mathrm{~nm}$.

\section{FBAI Passivation}

Benzylammonium iodide (BAI) and phenethylammonium iodine (PEAI) are successful passivation materials applied between perovskite/HTL interfaces ${ }^{24-27}$. Our previous research showed that chemically tailored passivation materials with different functional substituent groups can improve the photovoltaic performance and stability of PSCs simultaneously ${ }^{23} 28$. In this study we introduce a trifluoromethyl group at the para-position of the phenyl ring in BAI (Figure 1a), yielding FBAI as a new passivation material. Surface morphologies of the perovskites with and without FBAI passivation are characterized by SEM and the results are shown in Figure 1c and 1d. The slightly textured structure as observed in Figure 1d indicates formation of a top layer on the surface of the perovskite film, while the grain size does not change compared with the pristine perovskite film. Surface and depth X-ray photoelectron spectroscopy (XPS) profiling were employed to characterize the elemental distribution and chemical environments in the perovskite films with and without FBAI passivation $^{29}$, and the results are shown in Figure 2 and Figure S1. Pb 4f, F 1S and I 3d5 depth XPS profiling was used to determine the elemental distribution and thickness of the perovskite/FBAI film, and the thickness of the FBAI layer is estimated to be $\sim 5 \mathrm{~nm}$ (Figure 2a and $\mathbf{2 b}$ ). The binding energies of the surface elements are shown in Figure $2 \mathbf{c}$ and $\mathbf{2 d}$. A C $=\mathrm{O}$ peaks at $\sim 288 \mathrm{eV}$ is clearly observed for the pristine perovskite film, referring to the interaction between the moisture or oxygen in the air and the surface of the perovskite ${ }^{13}$. However, the absence of such a peak for perovskite/FBAI film indicates the FBAI passivation layer can effectively protect perovskite film from decomposition caused by water and oxygen under ambient conditions. The C-F and C-N peak appearing at the perovskite/FBAI film surface belong to the FBAI molecule itself. 
a

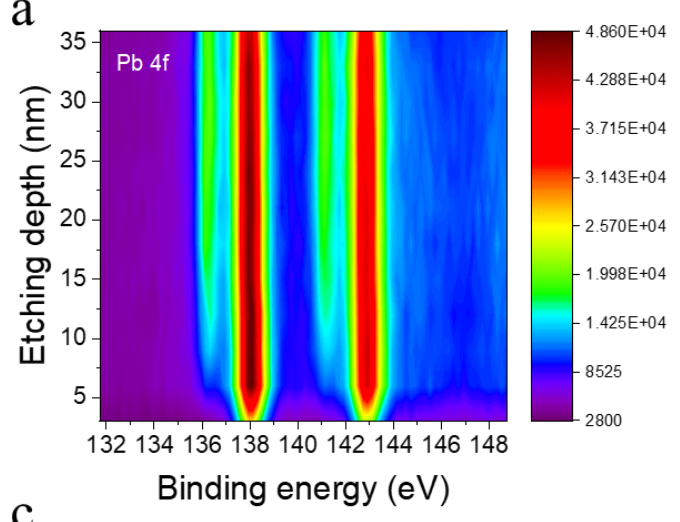

$\mathrm{C}$

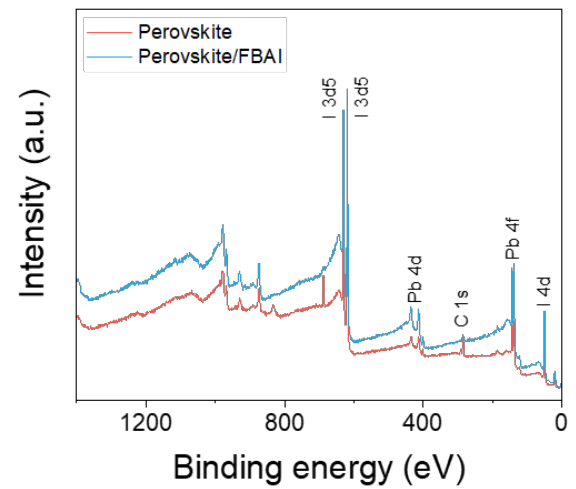

b

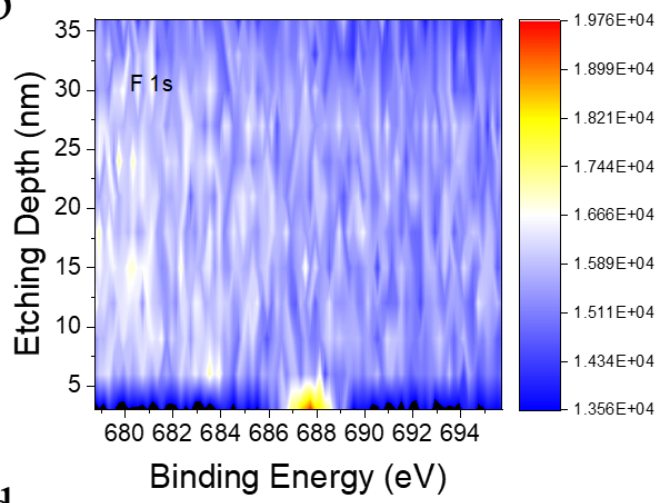

d

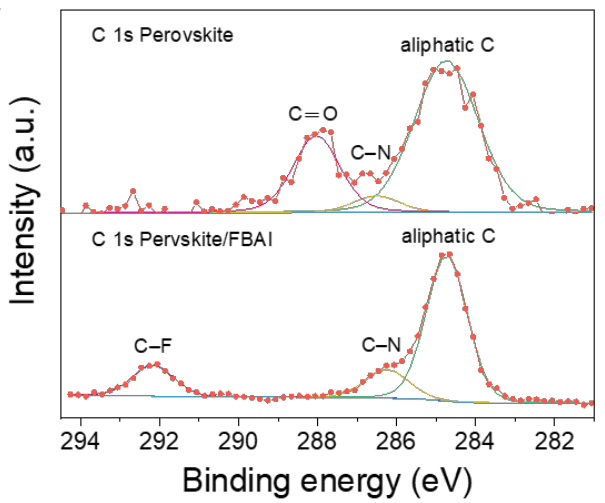

Figure 2. a-b) $\mathrm{Pb} 4 \mathrm{f}$ and $\mathrm{F}$ 1s XPS depth profiles of the perovskite/FBAI film. c) XPS survey spectra of neat perovskite and perovskite/FBAI films. d) High-resolution deconvoluted carbon 1s spectra for various films.

Structural information of the perovskite films with and without FBAI passivation is obtained by Xray diffraction (XRD) and the results are shown in Figure 3a. Compared with the pristine perovskite film, the diffraction peak corresponding to excess $\mathrm{PbI}_{2}$ for the FBAI treated perovskite disappeared, indicating that FBAI consumes the excess of lead iodide. Figure $\mathbf{3 b}$ shows the TRPL measurements of the perovskite film on $\mathrm{FTO} / \mathrm{Al}_{2} \mathrm{O}_{3}$ substrates with and without FBAI passivation. Since the bulk film is considered to have the same non-radiative recombination rate due to the identical perovskite active layer, we attribute the slower PL decay of the FBAI passivated film to a reduction in the surface recombination rate. To quantify this, we fitted the measured TRPL traces with the model described in Zhu et al. ${ }^{28}$, with the details of the simulations described in the Supporting Information (Supporting Note S1) and the results in Table S1. For both scenarios we obtain the same fit curves. As can be seen, FBAI passivation leads to a significant reduction of the surface recombination velocity of $115 \pm 25 \mathrm{~cm} \mathrm{~s}^{-1}$ indicating a significantly reduced surface defect density. The surface 
band structures with and without FBAI layer are measured with ultraviolet photoelectron spectroscopy (UPS) and the results are shown in Figure S2. The work function was determined to be $-4.15 \mathrm{eV}$ and $-4.42 \mathrm{eV}$ with the valance band maximum of $-5.46 \mathrm{eV}$ and $-5.68 \mathrm{eV}$ for perovskite and perovskite/FBAI film, respectively (Figure 3c). The deeper valence band bending for FBAI passivated perovskite allows to achieve a higher $V_{\mathrm{OC}}{ }^{30 ; 31}$.

a

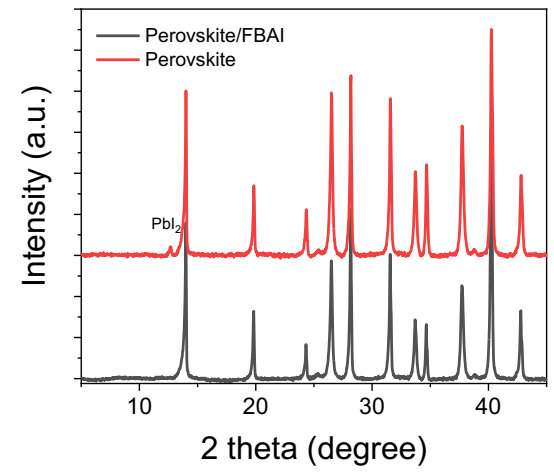

C

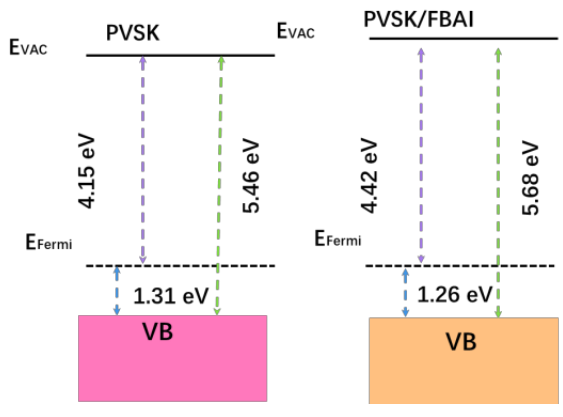

$\mathrm{b}$
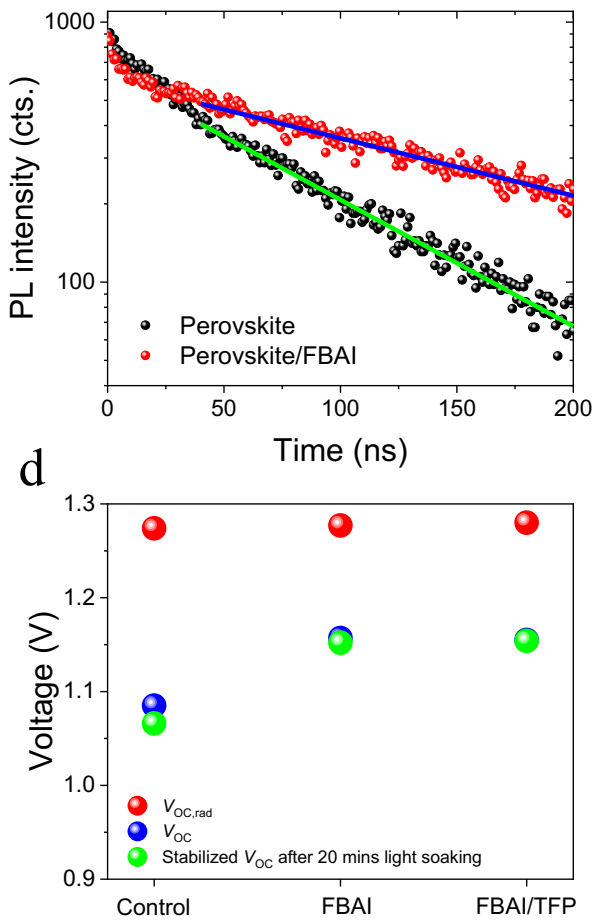

Figure 3. a) XRD patterns of neat perovskite and perovskite/FBAI films. b) TRPL decay and linear fit for glass/FTO/mesoscopic- $\mathrm{Al}_{2} \mathrm{O}_{3} /$ perovskite with and without $\mathrm{FBAI}$ passivation layer c) Energetic diagram for neat perovskite and perovskite/FBAI films. d) $V_{\mathrm{OC}}$ and radiative limit $V_{\mathrm{OC}}$ for glass/FTO/compact- $\mathrm{TiO}_{2} /$ mesoscopic- $\mathrm{TiO}_{2} /$ perovskite/ (with or without) interface layer/HTL /Au. The stabilized $V_{\mathrm{OC}}$ after 20 min light soaking is also shown.

PLQY $_{\text {ext }}$ is used to investigate charge recombination characteristics of perovskite and

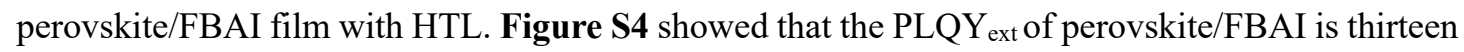
times higher than that of pristine film, implying that the FBAI layer can significantly suppress the non-radiative recombination at perovskite/spiro-OMeTAD interface. Voc losses of perovskite and perovskite/FBAI film with HTL were calculated by the equation: $\Delta V=k T \ln (P L Q Y)$. As shown 
in Figure 3d, the perovskite film with FBAI layer has a theoretical $V_{\mathrm{OC}}$ loss as small as $90 \mathrm{mV}$ compared to the counterpart pristine perovskite film exhibiting a $V_{\mathrm{OC}}$ loss of about $150 \mathrm{mV}$, allowing the device based on perovskite/FBAI to potentially afford a higher $V_{\mathrm{OC}}$. PSCs based on perovskite/spiro-OMeTAD, perovskite/FBAI/spiro-OMeTAD and perovskite/FBAI/spiroOMeTAD with TFP were fabricated and the radiative limits for $V_{\mathrm{OC}}\left(V_{\mathrm{oc}, \mathrm{rad}}\right){ }^{32}$ (the calculation method is shown in the Supporting Note S2) were compared using stabilized $V_{\mathrm{OC}}$ (Figure 3d). The smaller $V_{\mathrm{OC} \text {, rad }}$ and $V_{\mathrm{OC}}$ offset shows FBAI has an outstanding defect passivation capability when compared to PSC based on neat perovskite ${ }^{33}$. In addition, the $V_{\mathrm{OC}}$ drop for FBAI and TFP based PSC is significantly lower than that of pristine PSC after 20 mins light soaking, proving that FBAI passivated layer combined with TFP dopant can increase the short-term $V_{\mathrm{OC}}$ stability under light soaking in ambient air.

\section{Effect of TFP as HTL dopant}

The chemical structure of TFP is shown in Figure 1a. TFP is derived from $t \mathrm{BP}$ where the tert-butyl group on $t \mathrm{BP}$ is substituted by a trifluoromethyl group. The introduction of TFP into the HTL substantially increased the resistance to moisture uptake as revealed by contact angle measurements (CAMs). As shown in Figure S5, the contact angle between water droplet and HTL film with varying amounts of TFP (denoted as TFP $x$, where $x=0.1 \sim 0.6$ times the relative molar concentration of $t \mathrm{BP}$ added into the spiro-OMeTAD/tBP solution) increases from $70.5^{\circ}$ to $97.5^{\circ}$, indicating increased hydrophobicity by the substitution of $t$ BP by TFP. To verify the potential photovoltaic application of TFP in solar cell devices, PSC devices with a n-i-p structure of fluorine doped tin oxide (FTO)/compact $\mathrm{TiO}_{2} /$ mesoscopic $\mathrm{TiO}_{2} /$ perovskite/HTL/gold were fabricated, in which perovskite refers to a composition of $\mathrm{Cs}_{0.05} \mathrm{FA}_{0.85} \mathrm{MA}_{0.10} \mathrm{~Pb}\left(\mathrm{I}_{0.97} \mathrm{Br}_{0.03}\right)_{3}$ with $5 \%$ excess of $\mathrm{PbI}_{2}$, and various amounts of TFP are introduced into the HTL. The $I-V$ curves are shown in Figure S6, with summarized photovoltaic parameters shown in Table S2. Concentration screening shows that the best photovoltaic performances were obtained when $x=0.3$, TFP concentration at which the film shows good hydrophobicity with a contact angle up to $97.6^{\circ}$. Surface morphologies of the HTLs with and without TFP dopant are characterized by scanning electron microscope (SEM), and the results are shown in Figure S7. The negligible difference between the SEM images indicates that the introduction of TFP does not affect the film morphology during the casting of HTL. Hole- 
extraction and charge-recombination of the perovskite/HTLs with TFP are characterized by timeresolved photoluminescence (TRPL) and external photoluminescence quantum efficiency (PLQY ext) measurements ${ }^{34}$, with the later determined from the absolute intensity photon flux (AIPL) according to the methods reported by Mello et al. ${ }^{35}$, and the results are shown in Figure $\mathbf{S 8}$ and S9. Similar decay traces and PLQY ext are observed for HTL casted perovskite films with and without TFP, indicating that TFP neither compromises the extraction of holes nor alters the recombination process at perovskite/HTL interface.

\section{Photovoltaic Performance for PSC Employing TFP and FBAI}

In order to investigate the advantages of utilizing both FBAI and TFP in solar cell devices, PSCs were fabricated with a device architecture of fluorine-doped tin oxide (FTO)/compact$\mathrm{TiO}_{2} /$ mesoporous $\mathrm{TiO}_{2} /$ perovskite $(\mathrm{FBAI}) / \mathrm{HTL}$ (TFP)/gold. Figure 4a shows a cross-sectional SEM image of a PSC based on FBAI and TFP, with perovskite photo-absorber layer thickness of $\sim 400 \mathrm{~nm}$. $I-V$ curves of these cells under 1-sun illumination are shown in Figure $4 \mathbf{b}$, and the corresponding photovoltaic parameters are summarized in Table 1. The champion device based on perovskite/FBAI/TFP achieves a PCE of 23.94\%, with a $V_{\mathrm{OC}}$ of $1163 \mathrm{mV}$, an FF of 82.4\%, and a $J_{\mathrm{SC}}$ of $24.98 \mathrm{~mA} \mathrm{~cm}^{-2}$. Such a $V_{\mathrm{OC}}$ represents a voltage loss of $387 \mathrm{mV}$ with respect to the optical bandgap of $1.55 \mathrm{eV}$ (Figure $\mathbf{S 8}$ and Figure 3d). The $V_{\mathrm{OC}}$ of perovskite/FBAI/TFP based device is $64 \mathrm{mV}$ higher than that of the control device and is attributed to the significantly reduced nonradiative recombination at perovskite/spiro-OMeTAD interface by the FBAI layer, which is consistent with the $V_{\mathrm{OC}}$ loss calculated from PLQY. The device based on perovskite/FBAI/TFP obtains a negligible hysteresis of $0.6 \%$. In contrast, the hysteresis of the control device is $3.2 \%$, with $20.26 \%$ PCE for forward scan and $20.90 \%$ for reverse scan. Incident photon to electron conversion efficiency (IPCE) spectra are shown in Figure 4c, with the integrated current densities being 24.41 $\mathrm{mA} \mathrm{cm}^{-2}$ and $24.30 \mathrm{~mA} \mathrm{~cm}^{-2}$ for control PSC and PSC based on FBAI and TFP, respectively, which is in consistent with the $J_{\mathrm{SC}}$ obtain from $J-V$ measurements. Figure 4d shows the stabilized power output at the maximum power point (MPP) for the best performing devices under $10 \% r . h$. condition within the first $6 \mathrm{~min}$. For the perovskite/FBAI/TFP based device the stabilized PCE is estimated to be $23.83 \%$, which is in good agreement with the PCEs obtained from the forward and backward $J_{-}$ $V$ scans. 
a
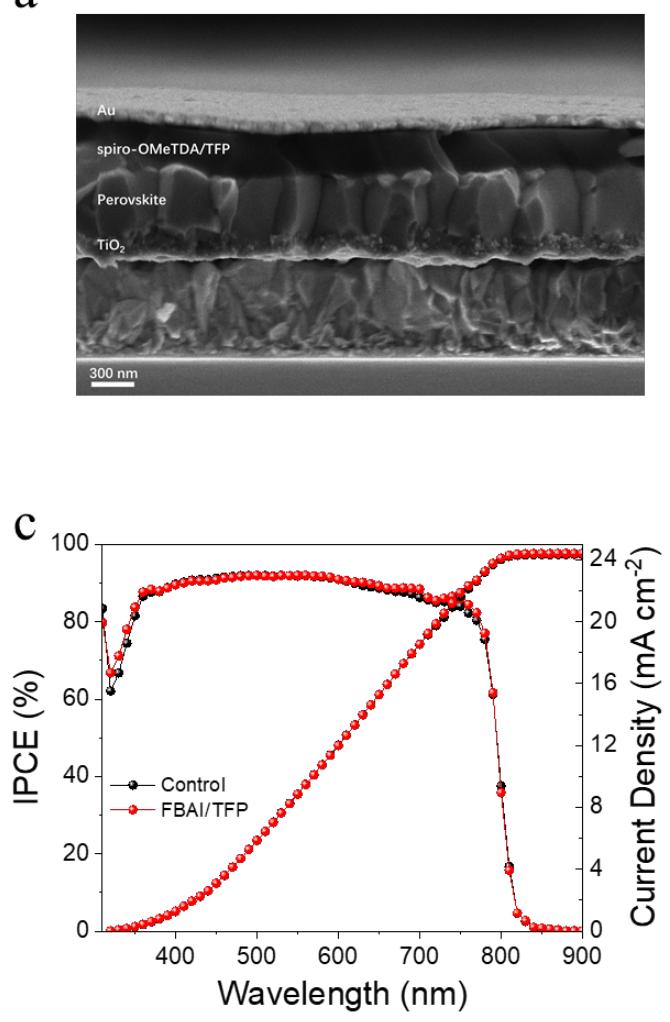

$\mathrm{b}$

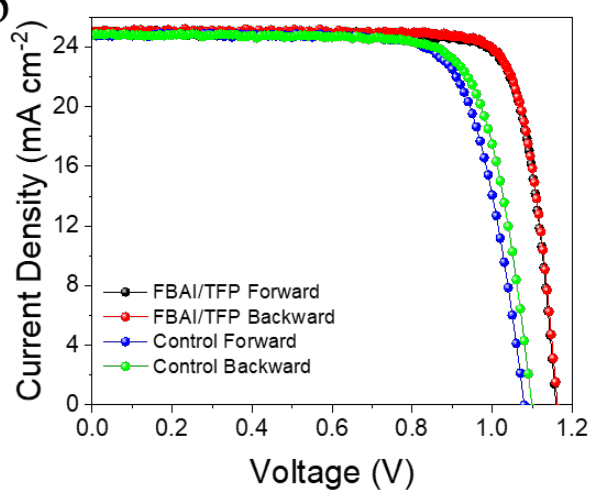

d

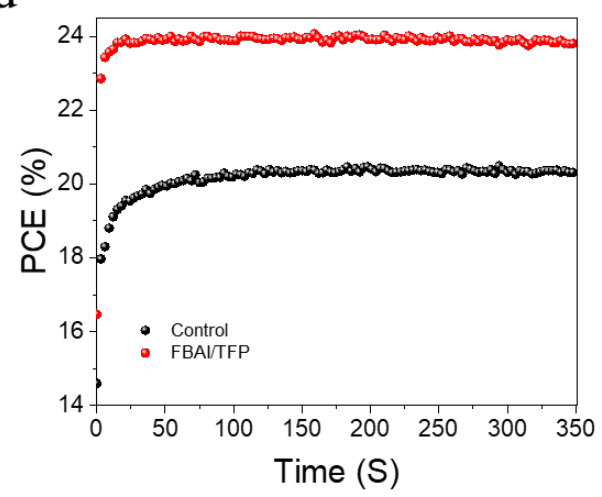

Figure 4. a) Cross-sectional SEM image of the PSC based on perovskite/FBAI/TFP. b) $I-V$ curves of the champion PSCs. c) IPCE and integrated current density values of the PSCs. d) MPP tracking of the PSCs within the first $330 \mathrm{~s}$ under ambient air.

We collected the photovoltaic parameters of 20 cells in one batch based on pristine perovskite and the other based on Perovskite/FBAI/TFP cells. As shown in the Figure 5a and Figure S10, the devices based on Perovskite/FBAI/TFP show superior reproducibility as compared to the control devices. The ideality factor $\left(\mathrm{n}_{\mathrm{id}}\right)$ of control PSC devices and PSC devises based on FBAI and TFP are calculated from the illumination intensity dependent $V_{\mathrm{OC}}$ measurements to investigate the recombination dynamics ${ }^{36}$, and the results are shown in Figure 5b. The $n_{\text {id }}$ of perovskite/FBAI/TFP is 1.57 , less than that of the control device $\left(n_{\text {id }}=1.86\right)$, better approaching a value of $n_{i d}=1$. The results indicate that perovskite/FBAI/TFP devices showed slower nonradiative recombination ${ }^{37}$, which is in good agreement with the improved photovoltaic performance for PSCs based on FBAI and TFP.

Table 1. Photovoltaic parameters of champion devices based on pristine perovskite and 
perovskite/FBAI/TFP (measured under simulated AM 1.5G irradiance).

\begin{tabular}{|c|c|c|c|c|c|c|}
\hline & $V_{\text {OC }}(V)$ & $\begin{array}{c}J_{S C^{a}} \\
\left(m A c^{-2}\right)\end{array}$ & $\begin{array}{c}J_{\mathrm{SC}}^{\mathrm{b}} \\
\left(\mathrm{mA} \mathrm{cm}{ }^{-2}\right)\end{array}$ & FF & $\begin{array}{r}\text { PCE } \\
(\%)\end{array}$ & $\begin{array}{c}\text { MPP-PCE } \\
(\%)\end{array}$ \\
\hline $\begin{array}{l}\text { FBAI/TFP } \\
\text { Backward }\end{array}$ & 1.163 & 24.98 & & 0.824 & 23.94 & \\
\hline $\begin{array}{c}\text { FBAI/TFP } \\
\text { Forward }\end{array}$ & 1.161 & 24.96 & 24.41 & 0.821 & 23.79 & 23.83 \\
\hline $\begin{array}{c}\text { Control } \\
\text { Backward }\end{array}$ & 1.099 & 24.78 & & 0.768 & 20.90 & \\
\hline $\begin{array}{l}\text { Control } \\
\text { Forward }\end{array}$ & 1.081 & 24.80 & 24.30 & 0.756 & 20.26 & 20.36 \\
\hline
\end{tabular}

a) $J_{\mathrm{SC}}$ determined from the IV measurement; b) $J_{\mathrm{SC}}$ determined from IPCE.

Operational and environmental stability of pristine, perovskite/FBAI and perovskite/FBAI/TFP devices are evaluated. Figure 5c and S11 show the operational stability results obtained with PSCs at maximum power point under 1 sun irradiation in nitrogen atmosphere $\left(\mathrm{N}_{2}\right)$. After $500 \mathrm{~h}$, the perovskite/FBAI/TFP based PSC retains $96.5 \%$ of its initial efficiency, in contrast to $91.2 \%$ for the PSC with only FBAI interlayer and 70\% for the pristine PSC. One of the reasons leading to better stability of the perovskite/FBAI/TFP based PSC devices results from a more stable FF $(\sim 97 \%$ compared to the control device of $\sim 63 \%$ ). This relatively stable FF value benefits from a more stable interface between perovskite and spiro-OMeTAD layer following passivation by $\mathrm{FBAI}^{28}$. Contact angle measurements of water droplets on the surface of perovskite films with and without FBAI interfacial layers are shown in Figure S12. The pristine perovskite and perovskite/FBAI film exhibit contact angles of $60.6^{\circ}$ and $99.3^{\circ}$, respectively. These results elucidate that FBAI functions by forming a hydrophobic layer on the perovskite surface ${ }^{38}$ to improve the stability of the perovskite. The devices based on Perovskite/FBAI/TFP show superior stability to those with FBAI passivation layer uniquely, due to an increase of hydrophobicity of spiro-OMeTAD layer (Figure S1) when TFP is used as additive. PSCs based on pristine, perovskite/FBAI and perovskite/FBAI/TFP devices were aged under ambient conditions with an r.h. of about $70 \%$, and the results are shown in Figure $\mathbf{5 d}$ and Figure S13. It is observed that after 45 days $(\approx 1100$ h), the PCE of PSC with perovskite/FBAI/TFP declined by less than $3 \%$, and PSC with the FBAI passivation layer only decreased by $5 \%$, while PSCs without interface layer decreased by over $40 \%$. The devices based on 
perovskite/FBAI/TFP achieve an excellent long-term stability indicating the synergistic effect of FBAI and TFP paves a new approach to improve PSC stability.
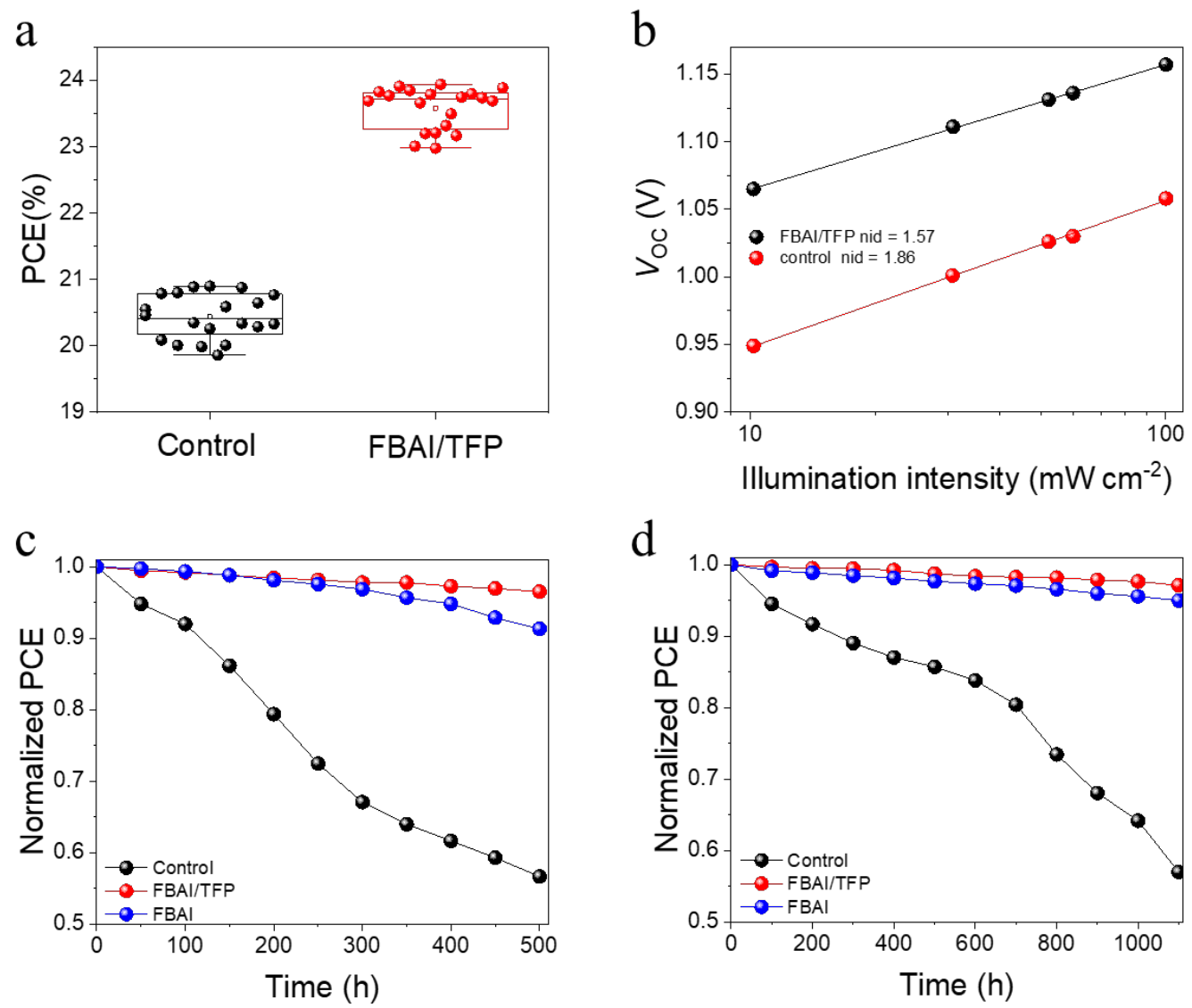

Figure 5. a) PCE distributions of 20 devices obtained from control and perovskite/FBAI/TFP device.

b) $V_{\mathrm{OC}}$ as a function of light intensity of control and perovskite/FBAI/TFP devices with ideality factors $\left(\mathrm{n}_{\mathrm{id}}\right)$. c) MPP ageing results in inert atmosphere $\left(\mathrm{N}_{2}\right)$ and under continuous 1 sun light soaking of pristine, perovskite/FBAI and perovskite/FBAI/TFP devices. d) Environmental stability of pristine, perovskite/FBAI and perovskite/FBAI/TFP device under a $r . h$. of about $60-70 \%$.

\section{Conclusion}

A new strategy to improve the photovoltaic performance and operational stability of PSCs has been demonstrated. Through the synergistic effect of trifluoromethylated passivation material (FBAI) and HTL dopant (TFP), we achieve an outstanding PCE approaching 24\% for PSCs with excellent longterm stability. XRD, TRPL and PLQY measurements reveal that the introduction of TFP can maintain the excellent hole extraction properties of the state-of-the-art spiro-OMeTAD/TBP/LiTFSI system, while increasing the hydrophobicity of the HTL film. FBAI-passivated perovskite films also exhibit significantly suppressed nonradiative charge carrier recombination at the 
perovskite/HTL interface and create a more moisture resistant interface. The champion cell based on perovskite/FBAI/TFP yielded a PCE of $23.9 \%$, which is considered among the highest efficiencies of mesoscopic PSCs so far. In addition, the enhanced hydrophobicity of the perovskite surface and HTL layer leads to a superior resistance to moisture uptake. Remarkably, PSCs with perovskite/FBAI/TFP retain over $96.5 \%$ of their initial PCE after $500 \mathrm{~h}$ MPP tracking under continuous full-sun light irradiation, and over $97 \%$ of their initial PCE after 45 days under ambient air with an $r . h$. of approximately $60-70 \%$. Our work provides an effective approach to achieve high efficiency and long-term operational stability for the advanced PSC technique.

\section{Acknowledgements}

The authors thank Dr. Carole Graetzel for her contribution to improve the quality of the languages and logic of the manuscript. H.Z. thanks the China Scholarship Council for funding. Y.L., S.M.Z., and M.G. thank the King Abdulaziz City for Science and Technology (KACST) and the European Union's Horizon 2020 research and innovation program (grant agreement No 826013) for financial support. X.L. acknowledges the financial support from the National Science Foundation of China (No. 21676188).

1. National Renewable Energy Laboratory Best Research-Cell Efficiency Chart (National Renewable Energy Laboratory Best Research-Cell Efficiency Chart, https://www.nrel.gov/pv/cellefficiency.html).

2. Zhou, H., Chen, Q., Li, G., Luo, S., Song, T.-b., Duan, H.-S., Hong, Z., You, J., Liu, Y., and Yang, Y. (2014). Interface engineering of highly efficient perovskite solar cells. Science 345, 542-546.

3. Wang, Y., Wu, T., Barbaud, J., Kong, W., Cui, D., Chen, H., Yang, X., and Han, L. (2019). Stabilizing heterostructures of soft perovskite semiconductors. Science 365, 687-691.

4. Min, H., Kim, M., Lee, S.-U., Kim, H., Kim, G., Choi, K., Lee, J.H., and Seok, S.I. (2019). Efficient, stable solar cells by using inherent bandgap of $\alpha$-phase formamidinium lead iodide. Science 366, 749-753.

5. Bai, S., Da, P., Li, C., Wang, Z., Yuan, Z., Fu, F., Kawecki, M., Liu, X., Sakai, N., Wang, J.T.-W., et al. (2019). Planar perovskite solar cells with long-term stability using ionic liquid additives. Nature 571, 245-250.

6. Wang, Z., Shi, Z., Li, T., Chen, Y., and Huang, W. (2017). Stability of Perovskite Solar Cells: A Prospective on the Substitution of the A Cation and X Anion. Angew. Chem., Int. Ed. 56, 11901212.

7. Rong, Y., Hu, Y., Mei, A., Tan, H., Saidaminov, M.I., Seok, S.I., McGehee, M.D., Sargent, E.H., and Han, H. (2018). Challenges for commercializing perovskite solar cells. Science 361, eaat8235.

8. Meng, X., Zhou, J., Hou, J., Tao, X., Cheung, S.H., So, S.K., and Yang, S. (2018). Versatility of 
Carbon Enables All Carbon Based Perovskite Solar Cells to Achieve High Efficiency and High Stability. Adv. Mater. 30, 1706975.

9. Hu, Q., Chen, W., Yang, W., Li, Y., Zhou, Y., Larson, B.W., Johnson, J.C., Lu, Y.-H., Zhong, W., $\mathrm{Xu}$, J., et al. (2020). Improving Efficiency and Stability of Perovskite Solar Cells Enabled by A NearInfrared-Absorbing Moisture Barrier. Joule 4, 1575-1593.

10. Chung, J., Shin, S.S., Kim, G., Jeon, N.J., Yang, T.-Y., Noh, J.H., and Seo, J. (2019). Impact of Electrode Materials on Process Environmental Stability of Efficient Perovskite Solar Cells. Joule 3, 1977-1985.

11. Kim, M., Kim, G.-H., Lee, T.K., Choi, I.W., Choi, H.W., Jo, Y., Yoon, Y.J., Kim, J.W., Lee, J., Huh,

D., et al. Methylammonium Chloride Induces Intermediate Phase Stabilization for Efficient Perovskite Solar Cells. Joule 3, 2179-2192.

12. Wang, Y., Zhang, T., Kan, M., Wang, X., Zhao, Y., Dar, M.I., Gratzel, M., Ono, L.K., Qi, Y., Li, Y., et al. (2019). Thermodynamically stabilized $\beta$-CsPbl3-based perovskite solar cells with efficiencies $>18$. Science 365, 591-595.

13. Jiang, Q., Zhao, Y., Zhang, X., Yang, X., Chen, Y., Chu, Z., Ye, Q., Li, X., Yin, Z., and You, J. (2019). Surface passivation of perovskite film for efficient solar cells. Nat. Photonics 13, 460-466.

14. Bu, T., Li, J., Zheng, F., Chen, W., Wen, X., Ku, Z., Peng, Y., Zhong, J., Cheng, Y.-B., and Huang, F. (2018). Universal passivation strategy to slot-die printed $\mathrm{SnO} 2$ for hysteresis-free efficient flexible perovskite solar module. Nat. Commun. 9, 1-10.

15. Zhang, F., Yao, Z., Guo, Y., Li, Y., Bergstrand, J., Brett, C.J., Cai, B., Hajian, A., Guo, Y., Yang, X., et al. (2019). Polymeric, Cost-Effective, Dopant-Free, Hole Transport Materials for Efficient and Stable Perovskite Solar Cells. J. Am. Chem. Soc. 141, 19700-19707.

16. Liao, H.-C., Tam, T.L.D., Guo, P., Wu, Y., Manley, E.F., Huang, W., Zhou, N., Soe, C.M.M., Wang, B., Wasielewski, M.R., et al. (2016). Dopant-Free Hole Transporting Polymers for High Efficiency, Environmentally Stable Perovskite Solar Cells. Adv. Energy Mater. 6, 1600502.

17. Yang, J., Liu, C., Cai, C., Hu, X., Huang, Z., Duan, X., Meng, X., Yuan, Z., Tan, L., and Chen, Y. (2019). High-Performance Perovskite Solar Cells with Excellent Humidity and Thermo-Stability via Fluorinated Perylenediimide. Adv. Energy Mater. 9, 1900198.

18. Kim, J.-E., Kim, S.-S., Zuo, C., Gao, M., Vak, D., and Kim, D.-Y. (2019). Humidity-Tolerant Rollto-Roll Fabrication of Perovskite Solar Cells via Polymer-Additive-Assisted Hot Slot Die Deposition. Adv. Funct. Mater. 10.1002/adfm.201809194,

19. Zhang, J., Zhang, T., Jiang, L., Bach, U., and Cheng, Y.-B. (2018). 4-tert-Butylpyridine Free Hole Transport Materials for Efficient Perovskite Solar Cells: A New Strategy to Enhance the Environmental and Thermal Stability. ACS Energy Lett. 3, 1677-1682.

20. Hawash, Z., Ono, L.K., and Qi, Y. (2018). Recent Advances in Spiro-MeOTAD Hole Transport Material and Its Applications in Organic-Inorganic Halide Perovskite Solar Cells. Adv. Mater. Interfaces 5, 1700623.

21. Schloemer, T.H., Christians, J.A., Luther, J.M., and Sellinger, A. (2019). Doping Strategies for Small Molecule Organic Hole-Transport Materials: Impacts on Perovskite Solar Cell Performance and Stability. Chem. Sci. 10.1039/c8sc05284k,

22. Jena, A.K., Ikegami, M., and Miyasaka, T. (2017). Severe Morphological Deformation of SpiroOMeTAD in (CH3NH3)Pbl3 Solar Cells at High Temperature. ACS Energy Lett. 10.1021/acsenergylett.7b00582,

23. Liu, Y., Akin, S., Uchida, R., Arora, N., Milic, J.V., Uhl, A.R., Zakeeruddin, S.M., Dar, M.I., Gratzel, 
M., Akin, S., et al. (2019). Ultrahydrophobic 3D/2D fluoroarene bilayer-based water-resistant perovskite solar cells with efficiencies exceeding 22. Sci. Adv. 5, eaaw2543.

24. Zhou, Y., Wang, F., Cao, Y., Wang, J.-P., Fang, H.-H., Loi, M.A., Zhao, N., and Wong, C.-P. (2017). Benzylamine-Treated Wide-Bandgap Perovskite with High Thermal-Photostability and Photovoltaic Performance. Adv. Energy Mater. 10.1002/aenm.201701048,

25. Gao, F., Zhao, Y., Zhang, X., and You, J. (2019). Recent Progresses on Defect Passivation toward Efficient Perovskite Solar Cells. Adv. Energy Mater. 10.1002/aenm.201902650,

26. Chen, B., Rudd, P.N., Yang, S., Huang, J., and Yuan, Y. (2019). Imperfections and their passivation in halide perovskite solar cells. Chem Soc Rev.

27. Liu, C., Masse, R., Jia, X., Bi, W., Neale, Z., Xu, M., Tian, M., Zheng, J., Cao, G., Yuan, J., et al. (2020). Interphases, Interfaces, and Surfaces of Active Materials in Rechargeable Batteries and Perovskite Solar Cells. Adv Mater, e1905245.

28. Zhu, H., Liu, Y., Eickemeyer, F.T., Pan, L., Ren, D., Ruiz-Preciado, M.A., Carlsen, B., Yang, B., Dong, X., Wang, Z., et al. (2020). Tailored Amphiphilic Molecular Mitigators for Stable Perovskite Solar Cells with 23.5\% Efficiency. Adv. Mater. 32, 1907757.

29. Wang, R., Xue, J., Meng, L., Lee, J.-W., Zhao, Z., Sun, P., Cai, L., Huang, T., Wang, Z., Wang, Z.K., et al. (2019). Caffeine Improves the Performance and Thermal Stability of Perovskite Solar Cells. Joule 3, 1464-1477.

30. Jiang, Q., Ni, Z., Xu, G., Lin, Y., Rudd, P.N., Huang, J., Xu, G., Xue, R., Li, Y., Li, Y., et al. (2020). Interfacial Molecular Doping of Metal Halide Perovskites for Highly Efficient Solar Cells. Adv Mater, e2001581.

31. Wang, R., Xue, J., Wang, K.-L., Wang, Z.-K., Luo, Y., Fenning, D., Xu, G., Nuryyeva, S., Huang, T., Zhao, Y., et al. (2019). Constructive molecular configurations for surface-defect passivation of perovskite photovoltaics. Science (Washington, DC, U. S.) 366, 1509-1513.

32. Krueckemeier, L., Rau, U., Stolterfoht, M., and Kirchartz, T. (2020). How to Report Record Open-Circuit Voltages in Lead-Halide Perovskite Solar Cells. Adv. Energy Mater. 10, 1902573.

33. Luo, D., Su, R., Zhang, W., Gong, Q., and Zhu, R. (2019). Minimizing non-radiative recombination losses in perovskite solar cells. Nat. Rev. Mater. 10.1038/s41578-019-0151-y,

34. Zhang, S., Hosseini, S.M., Gunder, R., Petsiuk, A., Caprioglio, P., Wolff, C.M., Shoaee, S., Meredith, P., Schorr, S., Unold, T., et al. (2019). The Role of Bulk and Interface Recombination in High-Efficiency Low-Dimensional Perovskite Solar Cells. Adv. Mater. 10.1002/adma.201901090,.

35. de Mello, J.C., Wittmann, H.F., and Friend, R.H. (1997). An improved experimental determination of external photoluminescence quantum efficiency. Adv. Mater. 9, 230-232.

36. Kong, X., Jiang, Y., Wu, X., Chen, C., Guo, J., Liu, S., Gao, X., Zhou, G., Liu, J.-M., Kempa, K., et al. (2020). Dopant-free F-substituted benzodithiophene copolymer hole-transporting materials for efficient and stable perovskite solar cells. J. Mater. Chem. A 8, 1858-1684.

37. Cai, M., Ishida, N., Li, X., Yang, X., Noda, T., Wu, Y., Xie, F., Naito, H., Fujita, D., and Han, L. (2018). Control of Electrical Potential Distribution for High-Performance Perovskite Solar Cells. Joule 10.1016/j.joule.2017.11.015, t.

38. Liu, Y., Akin, S., Hinderhofer, A., Eickemeyer, F.T., Zhu, H., Seo, J.-Y., Zhang, J., Schreiber, F., Zhang, H., Zakeeruddin, S.M., et al. (2020). Stabilization of Highly Efficient and Stable Phase-Pure FAPbl3 Perovskite Solar Cells by Molecularly Tailored 2D-Overlayers. Angew. Chem., Int. Ed. 10.1002/anie.202005211, 15688. 